\title{
Bridge-edges Mining in Complex Power Optical Cable Network based on Minimum Connected Chain Attenuation Topological Potential
}

\author{
Wanchang Jiang $^{1 *}$, Yanhui Liu ${ }^{1}$, Shengda Wang², and Jian Guo ${ }^{3}$ \\ ${ }^{1}$ School of Computer Science, Northeast Electric Power University \\ Jilin 132012, China \\ [e-mail: jwchang84@163.com, 1369442677@qq.com] \\ ${ }^{2}$ JiLin Information \& Telecommunication Company, Jilin Electric Power Corporation Ltd \\ Changchun 130021, China \\ [e-mail: dollie@126.com] \\ ${ }^{3}$ Jilin City Power Supply Bureau, Jilin Electric Power Corporation Ltd \\ Jilin 132011, China \\ [e-mail: 1680801@qq.com] \\ *Corresponding author: Wanchang Jiang
}

Received August 22, 2020; revised January 22, 2021; accepted February 27, 2021; published March 31, 2021

\begin{abstract}
The edges with "bridge characteristic" play the role of connecting the communication between regions in power optical cable network. To solve the problem of mining edges with "bridge characteristic" in provincial power optical cable network, the complex power optical cable network model is constructed. Firstly, to measure the generated potential energy of all nodes in n-level neighborhood local structure for one edge, the n-level neighborhood local structure topological potential is designed. And the minimum connected chain attenuation is designed to measure the attenuation degree caused by substituted edges. On the basis of that, the minimum connected chain attenuation topological potential based measurement is designed. By using the designed measurement, a bridge-edges mining algorithm is proposed to mine edges with "bridge characteristic". The experiments are conducted on the physical topology of the power optical cable network in Jilin Province. Compared with that of other three typical methods, the network efficiency and connectivity of the proposed method are decreased by $3.58 \%$ and $28.79 \%$ on average respectively. And the proposed method can not only mine optical cable connection with typical "bridge characteristic" but also can mine optical cables without obvious characteristics of city or voltage, but it have "bridge characteristic" in the topology structure.
\end{abstract}

Keywords: Complex Network, Power Optical Cable, Bridge-edge, Connected Chain Attenuation, Topological Potential 


\section{Introduction}

There are many complex systems in the real world, such as power network [1], traffic network [2], social science network [3], software system [4], etc. With the intention of study these systems, they are abstracted into complex networks, and the characteristics of robustness [5], vulnerability [6] and reliability [7] are studied. Especially, complexity research of the interconnected power network based on complex network has become one of the important research fields [8]. Cai et al. [9] shows that great achievements have been made in the theoretical research of vulnerability and self-organization criticality of the power grid.

In order to ensure the stable operation of power grid, the power communication network is produced [10]. And the network has been constructed by satellite, microwave, carrier, optical cable and other communication methods. As a supporting network, the power communication network is an important infrastructure of power grid [11]. With the gradual expansion of power communication network scale, the number of network nodes, network capacity and topology have undergone fundamental changes. At the same time, the requirements of power grid safety production present higher requirements for the reliability of power communication network [12].

At present, there are two aspects in the reliability research of power communication network based on complex network. On the one hand, the survivability of the network can be improved by mining and protecting key nodes. Liu et al. [13] improves the reliability by identifying and protecting key nodes of power communication network. And a protection strategy for high degree nodes and an edge adding strategy for low degree nodes are proposed to improve the structural vulnerability of power communication network in [14]. In reference [15], an evaluation strategy of node importance in power communication backbone network is proposed by using the node weight in power system as the correction coefficient. On the other hand, the reliability of power communication network is measured by link factor. He et al. [16] evaluated the link importance by considering the redundancy of network alternatives and the connectivity and non-connectivity of the network after link failure. On the basis of link used rate, the research method of the vulnerability of power communication network is put forward in [17]. Xia et al. [18] analyzes the affection of the topological structure on network reliability through calculation the importance of nodes and that of links.

In the study of bridge-edges and bridge-nodes, there are several scholars on bridge-edges or bridge-nodes in fields of dolphins' social network, email network and command and control network, etc. Considering the contribution of nodes to the importance of lines, a bridge-nodes mining method is proposed in [19]. Liu et al. [20] proposed a bridging node centrality method to identify bridging nodes. And the community structure is used to mine most influential nodes in [21]. These methods can only mine bridge-nodes, not bridge-edges. The edge betweenness [22] is a typical method to evaluate edges importance. However, global information is needed to calculate the shortest path of any pair of nodes. In large-scale networks, global information is not difficult to obtain. Pan et al. [23] proposed a bridge-edges mining measurement based on bridge coefficient. This method considers the size of degree and measures the influence of neighbor nodes on bridge-edges. However, it ignores the attenuation of "bridge characteristic" of edge caused by substitute edges, which replace the bridge-edge to connect the communication between regions. As the main communication mode of the power communication network, the role of edges with "bridge characteristic" in power optical cable network has not been discussed.

Therefore, in this paper, to measure the "bridge characteristic" of edges in power optical cable network, a complex power optical cable network model is constructed firstly. On the 
basis of topological potential, considering the attenuation caused by substitute edges to "bridge characteristic" of one edge, the minimum connected chain attenuation topological potential is proposed. Then, on the basis of that, a bridge-edges mining algorithm is designed to mine edges with "bridge characteristic" in provincial power optical cable network.

The rest of the paper is arranged as following. The second section introduces modeling of power optical cable network by using complex network theory. In the third section, the minimum connected chain attenuation topological potential is proposed to design bridge-edges measurement. The fourth section describes steps of bridge-edges mining measurement based on minimum connected chain attenuation topological potential. In the fifth section, we carry out two groups of experiments to verify the rationality and the validity of proposed method. The sixth section is the conclusion, including the future work.

\section{Complex Power Optical Cable Network Model}

As the dispatching, power plants and transformer substations in a power grid are connected by optical cables for communication, the physical network of power optical cable network has been constructed. According to complex network theory, the complex power optical cable network model $G=(V, E)$ is constructed to describe the physical network of provincial level, and following assumptions are made from the perspective of simplified analysis:

(1) Ignore the differences among each communication stations in provincial dispatching, standby dispatching, ground dispatching and power plants in the power optical cable network of provincial level, and abstract each communication station as one node with no difference;

(2) Ignore the length, number of cores and the voltage level of each optical cable link between two communication stations, and abstract the link as one edge with no weight and no direction;

(3) The double optical cable links between two communication stations are combined and regarded as one edge.

As a result, the complex power optical cable network model G can be constructed as follows:

$$
G=(V, E)
$$

where the node set $V=\left\{v_{i} \mid i=1,2 \cdots \cdots, N\right\}$, and $N$ is the number of nodes in $G$. The edge set $E=\left\{e_{i j} \mid i=1,2, \cdots \cdots, N, j=1,2, \cdots \cdots, N\right.$ and $\left.i \neq j\right\}$, and $M$ is the number of edges in $G$, where $e_{i j}=\left(v_{i}, v_{j}\right)$ is the edge from node $v_{i}$ to node $v_{j}$, and $e_{i j}=e_{j i}$.

The complex power optical cable network model of Jilin Province is shown in Fig. 1(a). Since power optical cable network has geographical topological connection, the communication stations in the same region form a regional power optical cable network. Due to the influence of geographical topology connection, the optical cable connection between regions is sparse, which leads to the emergence of edges with "bridge characteristic" in the power optical cable network.

This kind of edge usually plays an important role in communication between two regions. Simultaneously, take one local network in Fig. 1(a) as an example, it can be seen that there is one obvious bridge-edge for the local network with two regions, which is shown as Fig. 1(b). 


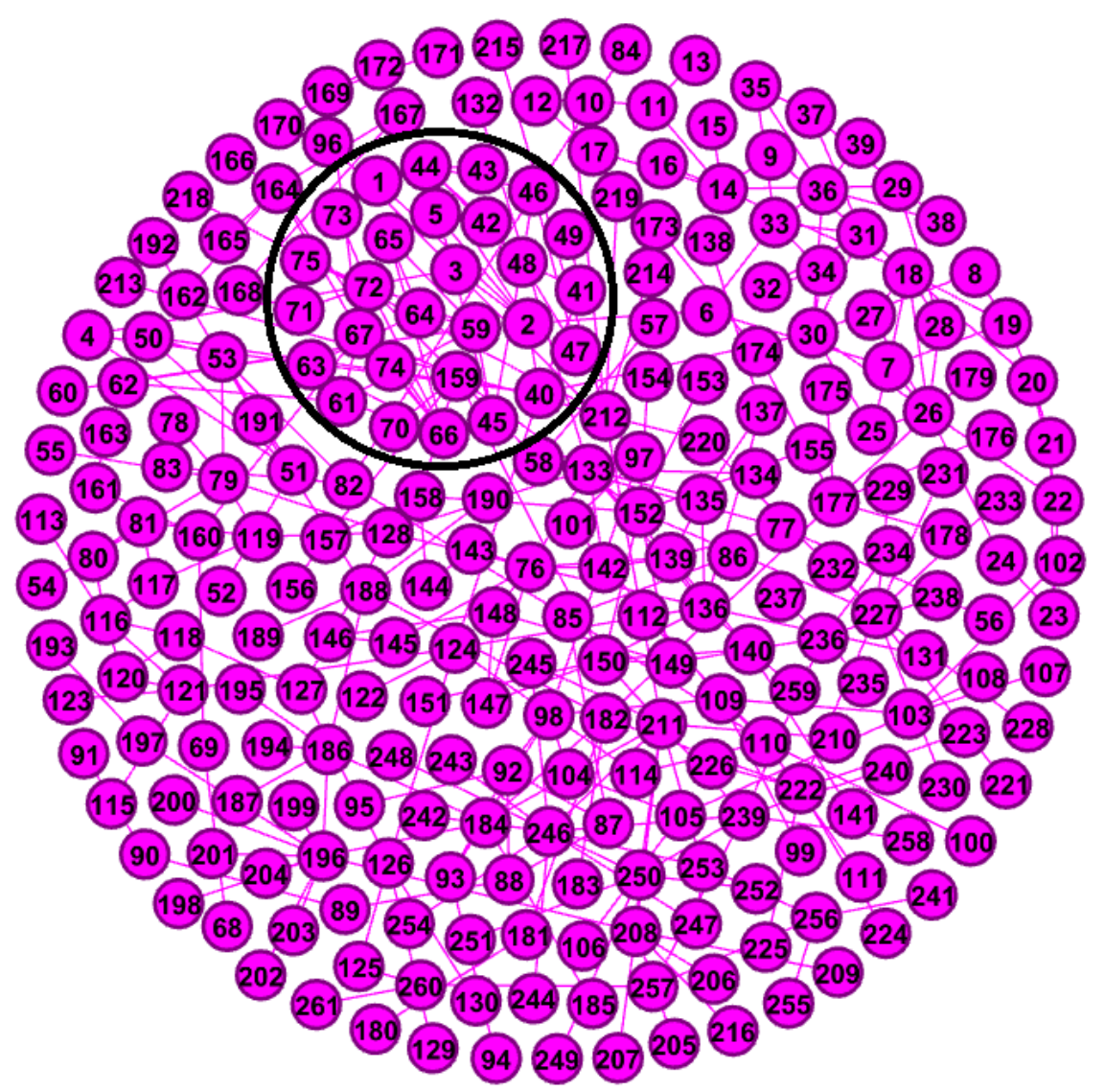

(a) The complex power optical cable network model

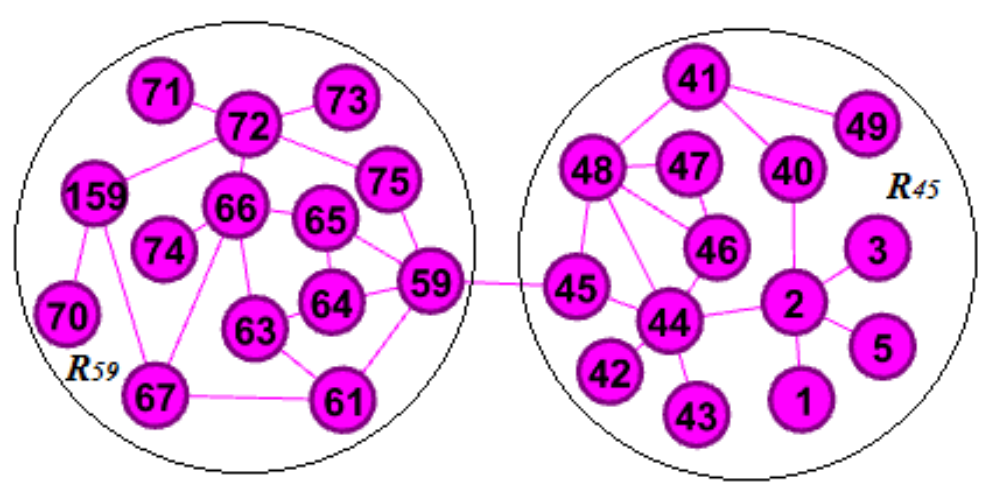

(b) One local network in the model

Fig. 1. The complex power optical cable network model of Jilin Province

As shown in Fig. 1(b), there is an edge $e_{45,59}$ with "bridge characteristic" that connecting two corresponding regions $R_{45}\left(e_{45,59}\right)$ and $R_{59}\left(e_{45,59}\right)$ in the local network, and communication between the two regions may be disconnected when the edge $e_{45,59}$ fails. Even if there are other paths to connect the two regions instead of the edge, the communication distance between the two regions will be greatly extended, which will lead to the decline of network efficiency. The edge of this kind is called "bridge-edge". 
In the power optical cable network, not all optical cable connections between $500 \mathrm{kV}$ communication stations and between municipal or county-level city are bridge-edges. And the optical cable without obvious characteristics such as city and voltage, but it has "bridge characteristic" in the topology structure, the optical cable is also the bridge-edge. Therefore, how to mine all bridge-edges of power optical cable network has an important impact on the reliability of the whole network.

\section{The Measurement of Bridge-edges Based on the Minimum Connected Chain Attenuation Topological Potential}

To measure the "bridge characteristic" of edges in one complex power optical cable network model, a novel bridge-edges measurement named minimum connected chain attenuation topological potential is designed by using the following four steps.

\section{1 n-level neighborhood local structure}

In order to describe the region that edge $e_{i j}$ can affect complex power optical cable network G, the n-level neighborhood local structure of edge $e_{i j}$ is defined.

Definition 1. (n-level neighborhood local structure): For each edge $e_{i j}$ in $G$, the n-level neighborhood local structure $G_{i j}$ is consist of edge $e_{i j}$, its two edge endpoints $v_{i}$ and $v_{j}$ and two corresponding n-level neighborhood regions $R_{i}\left(e_{i j}\right)$ and $R_{j}\left(e_{i j}\right)$.

Based on the shortest path length, the nodes in $G_{i j}$ close to the endpoint $v_{i}$ belong to $R_{i}\left(e_{i j}\right)$, and the nodes in $G_{i j}$ close to the endpoint $v_{j}$ belong to $R_{j}\left(e_{i j}\right)$.

Taking Fig. 1(b) as an example, it shows the 3-level $(n=3)$ neighborhood local structure of $e_{45,59}$. The 3-level neighborhood region of endpoint $v_{59}$ is $R_{59}\left(e_{45,59}\right)$, and the 3-level neighborhood region of endpoint $v_{45}$ is $R_{45}\left(e_{45,59}\right)$. Compared with endpoint $v_{45}$, node $v_{75}$ is closer to endpoint $v_{59}$, so node $v_{75}$ belongs to $R_{59}\left(e_{45,59}\right)$.

Note: only when the neighborhood local structure of one edge reaches n-level, the edge may have "bridge characteristic", and the edge will be put into the candidate bridge-edge set $\operatorname{con}_{k}(0<k<M)$ of network $G$.

\section{$3.2 \mathrm{n}$-level neighborhood local structure topological potential}

Classical topological potential can describe the interaction between nodes and the difference of topological positions. According to the theory of topological potential, one node in n-level neighborhood local structure $G_{i j}$ is regard as a potential source, which can affect other nodes and paths of connections among nodes in $G_{i j}$. Thus each node in $G_{i j}$ generates potential energy for $e_{i j}$, and these nodes interact with each other to form a virtual potential field in the network topology space. On the basis of that, n-level neighborhood local structure topological potential is designed, which is used to initially measure the "bridge characteristic" of edges in the candidate set $\operatorname{con}_{k}$.

Definition 2. (n-level neighborhood local structure topological potential): To measure the generated potential energy of all nodes in $G_{i j}$ for $e_{i j}$, the n-level neighborhood local structure topological potential is designed. It can be computed as follows:

$$
\varphi\left(e_{i j}\right)=\varphi\left(v_{i}\right)+\varphi\left(v_{j}\right)
$$


where $\varphi\left(v_{i}\right)$ is the topological potential of endpoint $v_{i} . \varphi\left(v_{i}\right)$ can be computed by the following formula :

$$
\varphi\left(v_{i}\right)=\sum_{s=1}^{N_{i s}}\left(m_{s} \times e^{-\left(\frac{d_{i s}}{\sigma}\right)^{2}}\right)
$$

where $d_{i s}$ is the shortest path from node $v_{i}$ to node $v_{s}$. The influence factor $\sigma$ is used to adjust the influence range of each node in the network. The $N_{i s}$ is the number of nodes in $R_{i}\left(e_{i j}\right)$. The $m_{s}$ represents the quality of node $v_{s}$, which can be used to describe the inherent attributes of node $v_{s}$.

$\varphi\left(v_{j}\right)$ is the topological potential of endpoint $v_{j} . \varphi\left(v_{j}\right)$ can be computed by the following formula :

$$
\varphi\left(v_{j}\right)=\sum_{h=1}^{N_{j h}}\left(m_{h} \times e^{-\left(\frac{d_{j h}}{\sigma}\right)^{2}}\right)
$$

where $d_{j h}$ is the shortest path from node $v_{j}$ to node $v_{h}$. The influence factor $\sigma$ is used to adjust the influence range of each node in the $G$. The $N_{j h}$ is the number of nodes in $R_{j}\left(e_{i j}\right)$. The $m_{h}$ represents the quality of node $v_{h}$, which can be used to describe the inherent attributes of node $v_{h}$.

\subsection{Minimum connected chain attenuation}

However, not all edges in the candidate set $\operatorname{con}_{k}$ are bridge-edges actually. For $G_{i j}$, if there is one substitute edge $e_{i, j}\left(i \neq i^{\prime}, j \neq j^{\prime}\right)$ that can replace $e_{i j}$ to connect $R_{i}\left(e_{i j}\right)$ and $R_{j}\left(e_{i j}\right)$, the edge $e_{i \prime j}$, will weaken the "bridge characteristic" of edge $e_{i j}$. All possible substitute edges for $e_{i j}$ are put into the substitute edge set $\left\{e_{i^{\prime} j^{\prime}}\right\}$ of $e_{i j}$, and an attenuation coefficient of "bridge characteristic" of edge $e_{i j}$ is designed.

Definition 3. (Minimum connected chain attenuation) : To measure the attenuation caused by substitute edges in $\left\{e_{i, j}\right\}$ the "bridge characteristic" of edge $e_{i j}$, the minimum connected chain attenuation is defined as a measurement of the attenuation degree of "bridge characteristic" of edge $e_{i j}$. The minimum connected chain attenuation of $e_{i j}$ is calculated as

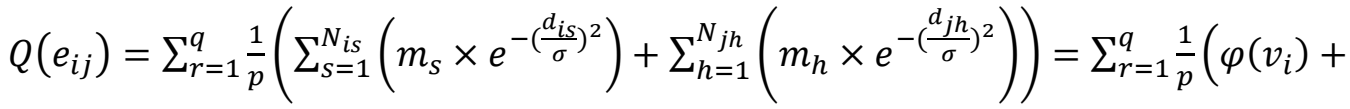

$$
\begin{aligned}
& \left.\varphi\left(v_{j}\right)\right)=\sum_{r=1}^{q} \frac{1}{p}\left(\varphi\left(e_{i j}\right)\right)
\end{aligned}
$$

where $q$ is the number of substitute edges in $\left\{e_{i, j},\right\}$. And edges $e_{i j}$ and $e_{i, j}$, can form multiple connected chains between $R_{i}\left(e_{i j}\right)$ and $R_{j}\left(e_{i j}\right)$, and the shortest one is taken as the minimum connected chain. And $p$ is the number of edges that make up the minimum connected chain. 


\subsection{Minimum connected chain attenuation topological potential based bridge- edges measurement}

The "bridge characteristic" of the edge $e_{i j}$ is attenuated due to the existence of substitute edges in $\left\{e_{i j^{\prime}}\right\}$. In this regard, a measurement is proposed to measure the value of "bridge characteristic" of the edge $e_{i j}$, which is called the minimum connected chain attenuation topological potential.

Definition 4. (Minimum connected chain attenuation topological potential): When substitute edge $e_{i, j}$, exists in $G_{i j}$ for $e_{i j}$, based on the preliminary measurement of degree value of "bridge characteristic" in the definition 2 and the attenuation coefficient in the definition 3, the minimum connected chain attenuation topological potential is designed to adjust the degree of "bridge characteristic" of the edge $e_{i j}$, which is taken as the final measurement of "bridge characteristic" of the edge $e_{i j}$.

$$
\begin{aligned}
& \operatorname{MCCA}\left(e_{i j}\right)=\sum_{s=1}^{N_{i s}}\left(m_{s} \times e^{-\left(\frac{d_{i s}}{\sigma}\right)^{2}}\right)+\sum_{h=1}^{N_{j h}}\left(m_{h} \times e^{-\left(\frac{d_{j h}}{\sigma}\right)^{2}}\right)-\sum_{r=1}^{q} \frac{1}{p}\left(\sum _ { s = 1 } ^ { N _ { i s } } \left(m_{s} \times\right.\right. \\
& \left.\left.e^{-\left(\frac{d_{i s}}{\sigma}\right)^{2}}\right)+\sum_{h=1}^{N_{j h}}\left(m_{h} \times e^{-\left(\frac{d_{j h}}{\sigma}\right)^{2}}\right)\right)=\varphi\left(e_{i j}\right)-Q\left(e_{i j}\right)
\end{aligned}
$$

After using the minimum connected chain attenuation, it can be seen that the larger $\varphi\left(e_{i j}\right)$ is and the smaller $Q\left(e_{i j}\right)$ is, which leads to the larger $\operatorname{MCCA}\left(e_{i j}\right)$, and makes the characteristic of edge $e_{i j}$ more pronounced. Namely, the "bridge characteristic" of the edge $e_{i j}$ between $v_{i}$ and $v_{j}$ is more obvious.

\section{Bridge-edges Mining Algorithm}

To mine edges with "bridge characteristic" in power optical cable network, a bridge-edges mining algorithm is proposed by using the minimum connected chain attenuation topological potential based "bridge characteristic" measurement. The algorithm is as follows:

\section{Algorithm MCCA}

Input: power optical cable network of provincial level

Output: bridge-edges

1. For each communication station $i(i=1,2, \cdots, \mathrm{N})$ do 2

2. Abstract communication station $i$ as node $v_{i}$;

3. For each optical cable link between station i and station $j(i \neq j)$ do 4

4. Abstract the optical cable link as edge $e_{i j}$;

5. Construct the complex power optical cable network model $G(V, E)$;

6. For each $v_{i}$ in $V$ do 7 to 10

7. Obtain the curve of $\mathrm{H}(\sigma)$ by changing $\sigma$;

8. Get the optimal value $\sigma^{\prime}$ corresponding to $H(\sigma)_{\min }$ from the curve;

9. Get the radius of $3 \sigma^{\prime} / \sqrt{2}$ hops by getting the corresponding $\sigma^{\prime}$;

10. The influence range of each node is determined as n-level by rounding $3 \sigma^{\prime} / \sqrt{2}$;

11. For each $e_{i j}$ in $\mathrm{E}$ do 12 to 13

12. Identify the n-level neighborhood local structure $G_{i j}$;

13. If the $G_{i j}$ of $e_{i j}$ reaches "n-level"

put the $e_{i j}$ into the candidate set $c o n_{k}$; 

14. For each $e_{i j}$ in con do $_{\mathrm{k}} 15$ to 17
15. Calculate the $\varphi\left(e_{i j}\right)$ by using formula (2);
16. Calculate the $Q\left(e_{i j}\right)$ by using formula (3);
17. Calculate $M C C A\left(e_{i j}\right)$ by using $\varphi\left(e_{i j}\right)$ and $Q\left(e_{i j}\right)$ with formula (4);
18. Rank all edges in $\operatorname{con}_{k}$ with $M C C A\left(e_{i j}\right)$ in descending sequence;
19. Obtain bridge-edges in power optical cable network.

The algorithm is a six-stage process. Steps 1 to 5 are the first stage, a complex power optical cable network model of provincial level power optical cable network is constructed.

In the second stage from step 6 to step 10, the influence range of each node is determined as $n$-level by rounding $3 \sigma^{\prime} / \sqrt{2}$, and the optimal value of the influence factor $\sigma^{\prime}$ corresponding to the minimum potential entropy $\mathrm{H}(\sigma)_{\min }$. The formula for $\mathrm{H}(\sigma)$ is as follows:

$$
H(\sigma)=-\sum_{i=1}^{n} \frac{\varphi\left(v_{i}\right)}{Z} \ln \left(\frac{\varphi\left(v_{i}\right)}{Z}\right)
$$

where the standardization factor $Z$ is $\sum_{i=1}^{n} \varphi\left(v_{i}\right)$.

In the third stage from steps 11 to 13 , the n-level neighborhood local structure of each edge is constructed and the edges that may have "bridge characteristic" are put into the bridge-edge candidate set $\operatorname{con}_{k}$.

In the fourth stage of steps 14 to 17 , considering the influence of the n-level neighborhood local structure and the substitute edge $e_{i \prime j}$, on the "bridge characteristic" of the edge $e_{i j}$, the minimum connected chain topological potential $\operatorname{MCCA}\left(e_{i j}\right)$ of $e_{i j}$ is calculated to measure the "bridge characteristic" of $e_{i j}$.

In the last part, from step 18 to step 19, all edges in the candidate set $\operatorname{con}_{k}$ are ranked in descending sequence with $\operatorname{MCCA}\left(e_{i j}\right)$. As a result, bridge-edges in the power optical cable network can be obtained.

\section{Experiments}

To verify the validity and rationality of the proposed bridge-edges mining algorithm based on minimum connected chain attenuation topological potential, two groups of experiments were conducted on two real world data sets, that is the physical topology of power optical cable network of the southern Jilin Province and the physical topology of power optical cable network of Jilin Province.

\subsection{Evaluation criteria}

In this paper, intentional attack is simulated on the mined bridge-edges in the complex power optical cable network model, the rationality and validity of the proposed method are verified by the following two evaluation criteria.

\subsubsection{Connectivity}

Connectivity refers to ratio of the number of nodes in the largest communication subset of network after the fault to the number of nodes in the network before the fault, which is an index to evaluate connectivity between communication nodes after fault [24], and any two 
nodes belonging to the largest communication subset can communicate with each other. The formula for connectivity is as follows:

$$
S=\frac{N^{\prime}}{N}
$$

where $\mathrm{N}^{\prime}$ is the number of nodes in the largest communication subset of the network after the fault; $\mathrm{N}$ is the number of nodes in the network before the fault.

The larger connectivity indicates that most nodes in network are still connected, and the stronger the communication ability of the network.

\subsubsection{Decline rates of network efficiency}

Network efficiency refers to the reciprocal sum of the path length between two nodes, which reflects the difficulty of information transmission in the network [15].

The definition of network efficiency $\eta$ is as follow:

$$
\eta=\frac{1}{N(N-1)} \sum_{v_{i} \neq v_{j} \in V} \eta_{i j}
$$

where the $\eta_{i j}$ is efficiency between $v_{i}$ and $v_{j}, \eta_{i j}=\frac{1}{d_{i j}}, d_{i j}$ is the shortest path between $v_{i}$ and $v_{j}$.

$\mu$ is the decline rate of network efficiency, defined as:

$$
\mu=1-\frac{\eta}{\eta_{0}}
$$

$\eta_{0}$ is the initial efficiency of the network.

The larger $\mu$ is, the greater damage caused by the attacked edge to network, indicating that the attacked edge is more important.

\subsection{Determination of the optimal value $\sigma^{\prime}$ in constructing the model}

When the potential energy reaches the minimum, the uncertainty of the whole topological potential field is the minimum. At the same time, the topological potential distribution is the most reasonable [25]. Therefore, the value of $\sigma^{\prime}$ corresponding to the minimum potential entropy $\mathrm{H}(\sigma)_{\min }$ is taken as the optimal value in the model. According to mathematical properties of Gauss function, the influence range of each node is a local region that is less than or equal to $3 \sigma^{\prime} / \sqrt{2}$ hops for given the optimal value of $\sigma^{\prime}$. When the distance is greater than $3 \sigma^{\prime} / \sqrt{2}$ hops, the unit potential function decays rapidly to 0 .

In order to mine bridge-edges, it is necessary to determine the optimal value $\sigma^{\prime}$ that corresponding to the minimum potential entropy $H(\sigma)_{\min }$. Then, the n-level neighborhood local structure $G_{i j}$ is constructed with the radius of $3 \sigma^{\prime} / \sqrt{2}$ hops. Fig. 2 shows the relationship between potential entropy $H(\sigma)$ and influence factor $\sigma$ of the southern network model, when the $\sigma^{\prime}$ is 1.8 , the potential entropy $H(\sigma)$ gets the minimum value. 


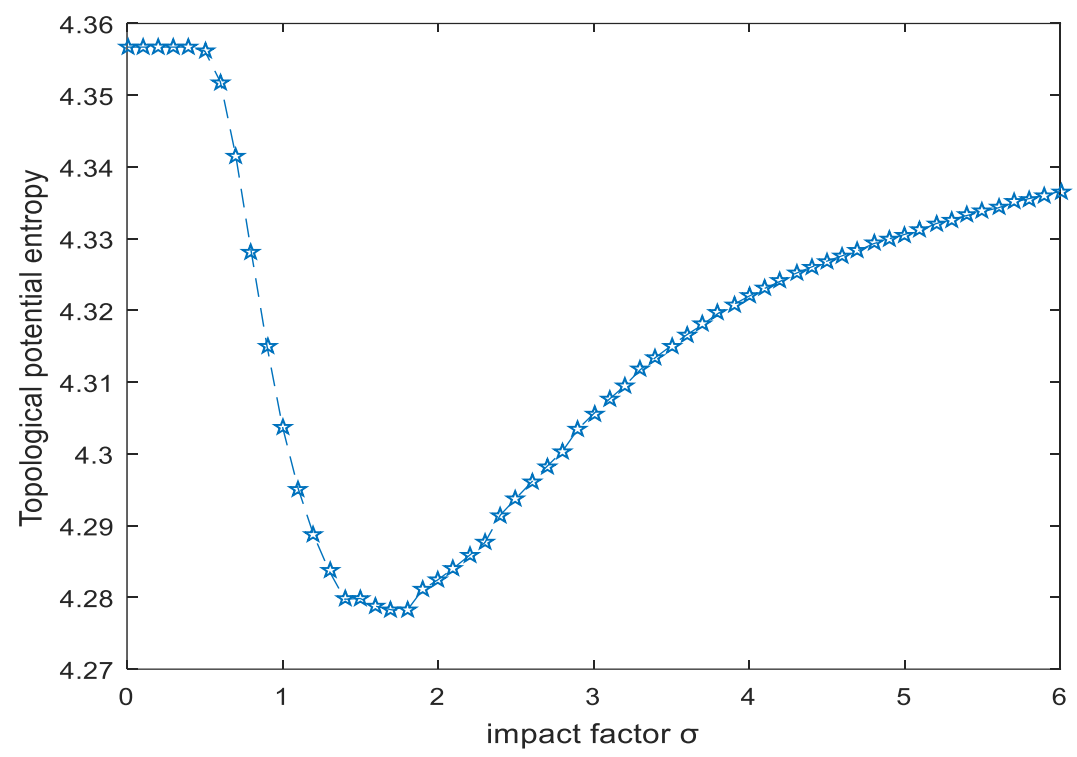

Fig. 2. Relationship between $H(\sigma)$ and $\sigma$ of the southern network

Fig. 3 shows the relationship between potential entropy $H(\sigma)$ and influence factor $\sigma$ of the provincial network model. When the $\sigma^{\prime}$ is still 1.8 , the potential entropy $H(\sigma)$ gets the minimum value.

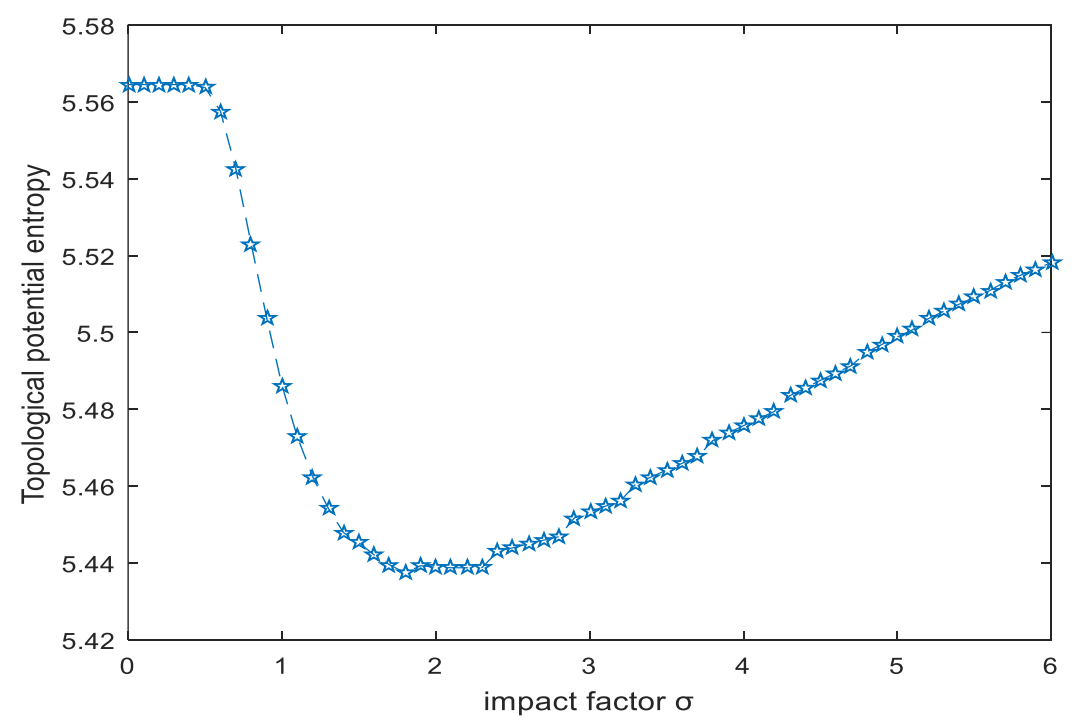

Fig. 3. Relationship between $\mathrm{H}(\sigma)$ and $\sigma$ of the provincial network in Jilin Province

When $\sigma^{\prime}$ is equal to 1.8 , the $3 \sigma^{\prime} / \sqrt{2}$ is equal to 3.82 . Based on the above analysis, the influence range of each node is a local region with the radius of 3.82 hops. When the distance is greater than 3.82 hops, the unit potential function quickly decays to 0 . Therefore, the influence range of each node is 3-level local region. Therefore, the $G_{i j}$ in the complex power optical cable network model is determined as 3-level. 


\subsection{Bridge-edges mining under four methods}

\subsubsection{Bridge-edges of the southern provincial network}

To illustrate the process of the proposed bridge-edges mining algorithm based on minimum connected chain attenuation topological potential, an example of the complex power optical cable network model of the southern network of Jilin Province is constructed, which is shown in Fig. 4. And the number of nodes is 78 and the number of edges is 114.

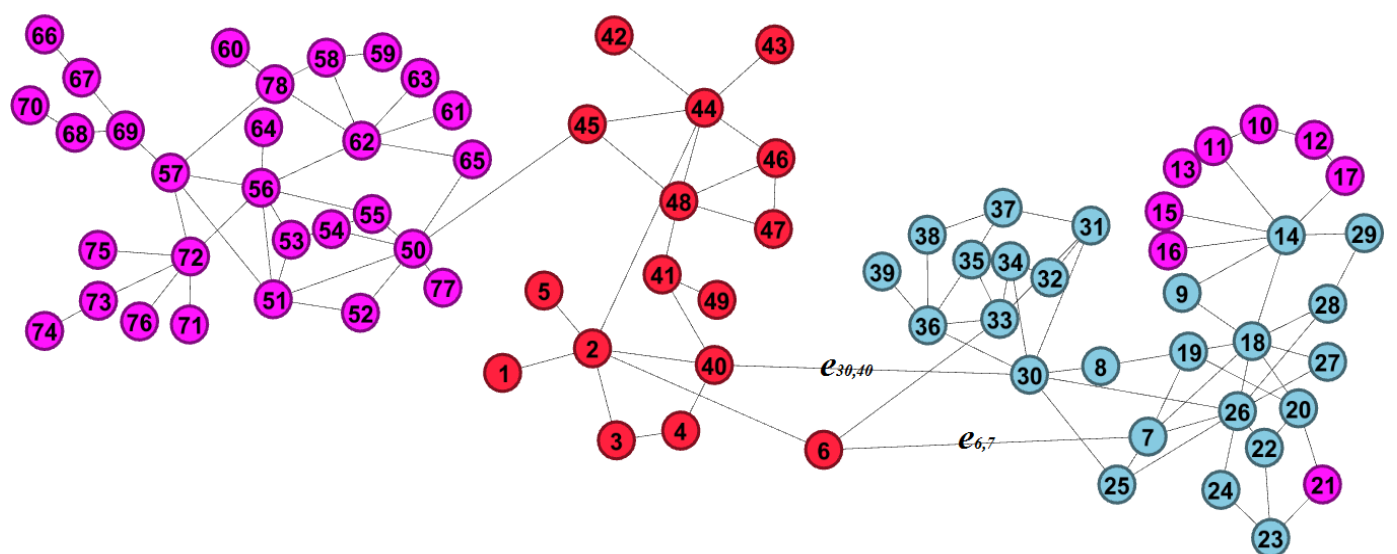

Fig. 4. The complex power optical cable network model of the southern Jilin Province

Take the edge $e_{30,40}$ in the model as an example, the blue part is the 3-level neighborhood region $R_{30}\left(e_{30,40}\right)$ of endpoint $v_{30}$ of $e_{30,40}$, and the red part is the 3-level neighborhood region $R_{40}\left(e_{30,40}\right)$ of endpoint $v_{40}$ of $e_{30,40}$. According to the definition 1 , the 3-level neighborhood local structure $G_{30,40}$ is constructed by edge $e_{30,40}$ and $R_{30}\left(e_{30,40}\right)$ and $R_{40}\left(e_{30,40}\right)$.

The topological potentials of endpoints $v_{30}$ and $v_{40}$ are 9.5 and 5.55 , respectively. Therefore, the n-level neighborhood local structure topological potential of edge $e_{30,40}$ is 15.05 by the definition 2 .

In $G_{30,40}$, there is one edge $e_{6,7}$ that can replace $e_{30,40}$ to connect $R_{30}\left(e_{30,40}\right)$ and $R_{40}\left(e_{30,40}\right)$, so $e_{30,40}$ and $e_{6,7}$ form a minimum connected chain between $R_{30}\left(e_{30,40}\right)$ and $R_{40}\left(e_{30,40}\right)$, which is composed of six edges. That is, $e_{30,40}, e_{40,2}, e_{2,6}, e_{6,7}, e_{7,25}, e_{25,30}$.

According to the definition 3, the minimum connected chain attenuation $Q\left(e_{30,40}\right)$ is 2.51 . Finally, the final "bridge characteristic" value $\operatorname{MCCA}\left(e_{30,40}\right)$ is 12.54 by the definition 4 . The calculation of each step of top $10 \%$ bridge-edges are shown in Table 1.

Table 1. The calculation of each step of top $10 \%$ bridge-edges

\begin{tabular}{|c|c|c|c|c|}
\hline order & edge & $\boldsymbol{\varphi}\left(\boldsymbol{e}_{\boldsymbol{i}, \boldsymbol{j}}\right)$ & $\mathrm{Q}\left(\boldsymbol{e}_{\boldsymbol{i}, \boldsymbol{j}}\right)$ & MCCA \\
\hline \hline 1 & $e_{30,40}$ & 15.05 & 2.51 & 12.54 \\
\hline 2 & $e_{45,50}$ & 11.59 & 0 & 11.59 \\
\hline 3 & $e_{6,7}$ & 12.76 & 2.12 & 10.64 \\
\hline 4 & $e_{2,6}$ & 12.69 & 2.11 & 10.58 \\
\hline 5 & $e_{2,44}$ & 12.19 & 2.43 & 9.76 \\
\hline
\end{tabular}




\begin{tabular}{|c|c|c|c|c|}
\hline 6 & $e_{40,41}$ & 10.53 & 2.10 & 8.43 \\
\hline 7 & $e_{56,72,}$ & 11.87 & 3.95 & 7.92 \\
\hline 8 & $e_{51,57}$ & 11.13 & 3.71 & 7.42 \\
\hline 9 & $e_{26,30}$ & 15.64 & 8.34 & 7.30 \\
\hline 10 & $e_{26,28}$ & 10.84 & 3.61 & 7.23 \\
\hline 11 & $e_{56,62}$ & 13.09 & 5.89 & 7.20 \\
\hline 12 & $e_{41,48}$ & 8.87 & 1.77 & 7.10 \\
\hline 13 & $e_{57,72}$ & 10.56 & 3.52 & 7.04 \\
\hline 14 & $e_{44,45}$ & 10.06 & 3.35 & 6.71 \\
\hline 15 & $e_{55,56}$ & 11.80 & 5.90 & 5.90 \\
\hline
\end{tabular}

For the southern provincial network model, the minimum connected chain attenuation topological potential (MCCA), degree summation (DS), bridge coefficient (BC) and edge betweenness (EB) are used to compare the mined results of bridge-edges. The top 5\% and top $10 \%$ bridge-edges results under four methods are shown in Table 2.

Table 2. The top 5\% and 10\% bridge-edges results under four methods

\begin{tabular}{|c|c|c|c|c|c|c|c|c|}
\hline order & edge & MCCA & edge & EB & edge & DS & edge & BC \\
\hline \hline 1 & $e_{30,40}$ & 12.54 & $e_{45,50}$ & 1292.8 & $e_{18,26}$ & 16 & $e_{18,26}$ & 63.9 \\
\hline 2 & $e_{45,50}$ & 11.59 & $e_{2,44}$ & 1094.4 & $e_{14,18}$ & 15 & $e_{14,18}$ & 56.0 \\
\hline 3 & $e_{6,7}$ & 10.64 & $e_{44,45}$ & 1018.6 & $e_{26,30}$ & 15 & $e_{26,30}$ & 56.0 \\
\hline 4 & $e_{2,6}$ & 10.58 & $e_{2,6}$ & 847.3 & $e_{7,18}$ & 13 & $e_{56,62}$ & 42.0 \\
\hline 5 & $e_{2,44}$ & 9.76 & $e_{6,7}$ & 688.9 & $e_{7,26}$ & 13 & $e_{56,72}$ & 42.0 \\
\hline 6 & $e_{40,41}$ & 8.43 & $e_{50,51}$ & 617.1 & $e_{56,62}$ & 13 & $e_{7,18}$ & 39.9 \\
\hline 7 & $e_{56,72}$ & 7.92 & $e_{14,18}$ & 549.4 & $e_{56,72}$ & 13 & $e_{7,26}$ & 39.8 \\
\hline 8 & $e_{51,57}$ & 7.42 & $e_{30,40}$ & 536.7 & $e_{2,44}$ & 12 & $e_{2,44}$ & 36.0 \\
\hline 9 & $e_{26,30}$ & 7.30 & $e_{7,18}$ & 499.9 & $e_{18,19}$ & 12 & $e_{30,36}$ & 34.9 \\
\hline 10 & $e_{26,28}$ & 7.23 & $e_{51,57}$ & 454.5 & $e_{18,20}$ & 12 & $e_{50,51}$ & 34.9 \\
\hline 11 & $e_{56,62}$ & 7.20 & $e_{26,30}$ & 384.2 & $e_{30,36}$ & 12 & $e_{51,56}$ & 34.9 \\
\hline 12 & $e_{41,48}$ & 7.10 & $e_{57,69}$ & 364.0 & $e_{50,51}$ & 12 & $e_{56,57}$ & 34.9 \\
\hline 13 & $e_{57,72}$ & 7.04 & $e_{50,65}$ & 355.9 & $e_{51,56}$ & 12 & $e_{18,19}$ & 31.9 \\
\hline 14 & $e_{44,45}$ & 6.71 & $e_{62,65}$ & 317.6 & $e_{56,77}$ & 12 & $e_{18,20}$ & 31.9 \\
\hline 15 & $e_{55,56}$ & 5.90 & $e_{40,41}$ & 284.4 & $e_{18,28}$ & 11 & $e_{57,72}$ & 29.9 \\
\hline
\end{tabular}

From the mined the top 5\% bridge-edges in Table 2, it can be found that when the top 5\%( the grey part) bridge-edges mined by MCCA are broken, the southern provincial network is divided into three connected subgraphs. However, when the top 5\% bridge-edges mined by other three methods are broken, the network is divided into two connected sub-networks at most. From the mined the top $10 \%$ bridge-edges, it can be found that edges $e_{30,40}, e_{45,50}$, $e_{2,44}, e_{2,6}, e_{6,7}, e_{56,72}, e_{40,41}, e_{56,62}, e_{44,45}, e_{57,72}$ are mined two or more times under four different methods. Meanwhile, the edge $e_{30,40}$ is optical cable connection between Tonghua $500 \mathrm{kV}$ communication station and Dongfeng $500 \mathrm{kV}$ communication station. The edge $e_{2,44}$ is the optical cable connection with "bridge characteristic" between the city of Meihe communication station and the city of Liaoyuan communication station, and the edge is mined by all methods at the same time. 


\subsubsection{Bridge-edges of the whole provincial network}

The complex power optical cable network model was constructed based on the physical topology of power optical cable network of Jilin Province, which is shown in Fig. 1(a). The number of nodes is 261 and the number of edges is 353. And the top 5\% and the top $10 \%$ bridge-edges results of DS, BC, EB and MCCA are shown in Table 3.

Table 3. The top 5\% and $10 \%$ bridge-edges results of DS, BC, EB and MCCA

\begin{tabular}{|c|c|c|c|c|c|c|c|c|}
\hline order & edge & EB & edge & MCCA & edge & DS & edge & BC \\
\hline $\bar{~} 1$ & $e_{136,150}$ & 12496.93 & $\boldsymbol{e}_{124,136}$ & 19.89 & $e_{211,246}$ & 21 & $e_{211,246}$ & 109.91 \\
\hline 2 & $e_{124,136}$ & 12440.58 & $e_{40,124}$ & 18.93 & $e_{150,211}$ & 19 & $e_{150,211}$ & 89.96 \\
\hline 3 & $e_{150,211}$ & 9123.75 & $e_{152,227}$ & 18.27 & $e_{196,208}$ & 19 & $e_{196,208}$ & 87.98 \\
\hline 4 & $e_{40,124}$ & 8936.68 & $e_{184,211}$ & 17.20 & $e_{239,246}$ & 19 & $e_{211,246}$ & 87.92 \\
\hline 5 & $e_{30,40}$ & 6584.29 & $e_{67,159}$ & 16.91 & $e_{76,136}$ & 18 & $e_{136,150}$ & 80.97 \\
\hline 6 & $e_{114,124}$ & 6080.28 & $e_{30,40}$ & 16.50 & $\boldsymbol{e}_{136,150}$ & 18 & $e_{76,136}$ & 80.87 \\
\hline 7 & $e_{110,114}$ & 5884.48 & $e_{196,208}$ & 16.07 & $e_{152,227}$ & 18 & $e_{152,227}$ & 80.00 \\
\hline 8 & $e_{150,159}$ & 5428.83 & $e_{222,227}$ & 15.40 & $e_{150,152}$ & 18 & $e_{211,212}$ & 80.00 \\
\hline 9 & $e_{184,211}$ & 5327.17 & $e_{150,211}$ & 15.29 & $e_{211,212}$ & 18 & $e_{150,152}$ & 79.95 \\
\hline 10 & $e_{67,159}$ & 4201.58 & $e_{236,239}$ & 15.23 & $e_{211,239}$ & 18 & $e_{211,239}$ & 79.9 \\
\hline 11 & $e_{26,30}$ & 3775.37 & $\boldsymbol{e}_{182,222}$ & 14.94 & $e_{246,250}$ & 18 & $e_{246,250}$ & 76.90 \\
\hline 12 & $e_{109,110}$ & 3719.06 & $e_{121,124}$ & 14.69 & $e_{76,133}$ & 17 & $\boldsymbol{e}_{150,159}$ & 71.95 \\
\hline 13 & $e_{182,184}$ & 2998.48 & $e_{124,126}$ & 14.49 & $e_{133,136}$ & 17 & $e_{150,152}$ & 71.94 \\
\hline 14 & $e_{152,227}$ & 2959.00 & $\boldsymbol{e}_{150,159}$ & 13.12 & $e_{150,152}$ & 17 & $e_{150,239}$ & 71.94 \\
\hline 15 & $e_{150,152}$ & 2354.63 & $e_{182,184}$ & 12.82 & $e_{150,159}$ & 17 & $e_{76,133}$ & 71.86 \\
\hline 16 & $e_{208,211}$ & 2278.85 & $e_{77,139}$ & 12.30 & $e_{150,239}$ & 17 & $e_{133,136}$ & 71.84 \\
\hline 17 & $e_{18,26}$ & 2129.10 & $e_{40,41}$ & 12.23 & $e_{186,196}$ & 17 & $e_{211,250}$ & 69.88 \\
\hline 18 & $e_{150,239}$ & 2070.16 & $e_{208,250}$ & 12.00 & $e_{211,250}$ & 17 & $e_{186,196}$ & 65.98 \\
\hline 19 & $e_{14,18}$ & 2010.17 & $e_{140,236}$ & 11.69 & $e_{18,26}$ & 16 & $e_{152,159}$ & 63.93 \\
\hline 20 & $e_{211,212}$ & 1838.51 & $e_{45,59}$ & 11.66 & $e_{76,139}$ & 16 & $e_{18,26}$ & 63.90 \\
\hline 21 & $e_{85,110}$ & 1780.10 & $e_{147,150}$ & 11.48 & $e_{136,139}$ & 16 & $e_{76,139}$ & 62.84 \\
\hline 22 & $e_{177,182}$ & 1777.00 & $e_{53,79}$ & 11.45 & $e_{152,159}$ & 16 & $e_{136,139}$ & 62.82 \\
\hline 23 & $e_{121,124}$ & 1741.45 & $e_{2,6}$ & 11.43 & $e_{184,196}$ & 16 & $e_{222,227}$ & 60.00 \\
\hline 24 & $e_{2,40}$ & 1721.68 & $e_{182,188}$ & 11.32 & $e_{222,227}$ & 16 & $e_{14,18}$ & 55.96 \\
\hline 25 & $e_{222,227}$ & 1601.85 & $e_{186,188}$ & 11.11 & $e_{14,18}$ & 15 & $e_{26,30}$ & 55.96 \\
\hline 26 & $e_{85,87}$ & 1564.59 & $e_{6,7}$ & 10.74 & $e_{26,30}$ & 15 & $e_{208,250}$ & 55.94 \\
\hline 27 & $e_{2,44}$ & 1508.64 & $e_{109,110}$ & 10.73 & $e_{124,136}$ & 15 & $e_{239,250}$ & 55.89 \\
\hline 28 & $e_{103,109}$ & 1499.00 & $\boldsymbol{e}_{136,150}$ & 10.70 & $e_{133,139}$ & 15 & $e_{133,139}$ & 55.80 \\
\hline 29 & $e_{98,109}$ & 1403.89 & $e_{208,211}$ & 10.64 & $e_{184,211}$ & 15 & $e_{184,196}$ & 54.96 \\
\hline 30 & $e_{45,59}$ & 1355.40 & $e_{2,44}$ & 10.57 & $e_{208,250}$ & 15 & $e_{124,136}$ & 54.00 \\
\hline 31 & $e_{184,186}$ & 1353.83 & $e_{186,196}$ & 10.57 & $e_{239,250}$ & 15 & $\boldsymbol{e}_{184,211}$ & 49.98 \\
\hline 32 & $e_{67,164}$ & 1338.37 & $e_{58,77}$ & 10.16 & $e_{246,253}$ & 15 & $e_{67,159}$ & 47.97 \\
\hline 33 & $e_{184,196}$ & 1310.85 & $e_{40,50}$ & 9.95 & $e_{67,159}$ & 14 & $e_{236,239}$ & 47.97 \\
\hline 34 & $e_{124,126}$ & 1299.77 & $e_{61,67}$ & 9.85 & $e_{72,159}$ & 14 & $e_{72,159}$ & 47.95 \\
\hline 35 & $e_{72,159}$ & 1282.37 & $e_{126,127}$ & 9.75 & $e_{76,128}$ & 14 & $e_{76,128}$ & 44.91 \\
\hline
\end{tabular}

From the mined bridge-edges of the four methods in Table 3, the similarity rates of mined bridge-edges of MCCA with that of EB, DS and BC are 51.43\% (18), 34\% (11) and 37\% (13), 
respectively. In addition, the bridge-edges $e_{182,222}, e_{77,139}, e_{53,79}, e_{6,7} e_{182,188}, e_{40,50}$, $e_{186,188}, e_{2,6}, e_{147,150}, e_{58,77}, e_{61,67}, e_{126,127}$ are only mined by MCCA, which are optical cable connections with "bridge characteristic" between county-level communication stations. As shown in the bold edges in the table, $77 \%$ (10) of optical cable connections between $500 \mathrm{kV}$ communication stations are mined by EB and MCCA, and 46\% (6) are mined by DS and BC. In the top 5\% of bridge-edges, 70\% (9) of optical cable connections between $500 \mathrm{kV}$ communication stations are mined by EB and MCCA, and 23\% (3) are mined by DS and BC.

\subsubsection{Network connectivity analysis}

For the southern provincial network and the provincial network, intentional attack is simulated on the bridge-edges mined by DS, BC, EB and MCCA, and the connectivity of the network may be destroyed.

\section{A. Connectivity on the southern provincial network}

Fig. 5 shows the trend of connectivity with the removal of bridge-edges in the southern complex power optical cable network model.

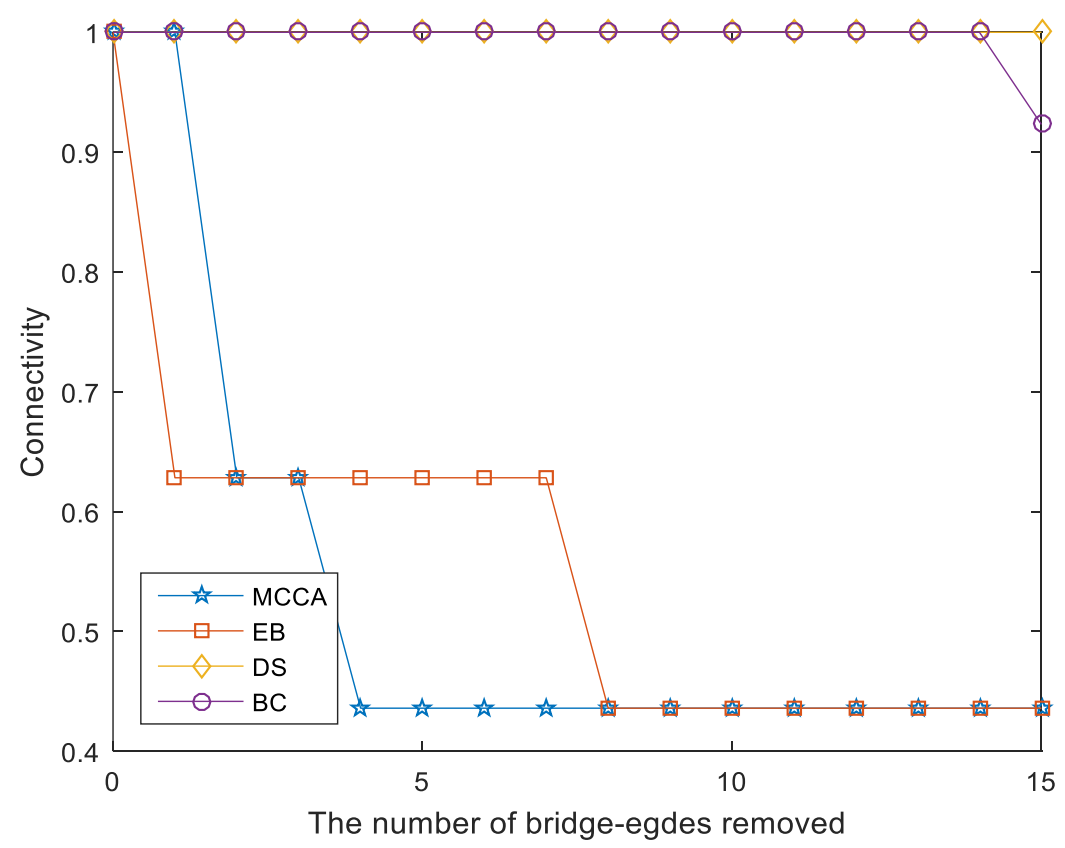

Fig. 5. The trend of network connectivity with the removal of bridge-edges

In Fig. 5, the curve of network connectivity of the four methods shows a downward trend as a whole. When the first one bridge-edge is removed from EB and the first two bridge-edges are removed from MCCA, the network is divided into two independent sub-networks, and the connectivity of both MCCA and EB are decreased by $37.18 \%$. When the first four edges are removed, as show in Fig. 6, the network of the MCCA method is divided into three independent sub-networks, and the connectivity of MCCA is decreased by $19.23 \%$, while the connectivity of EB does not decrease. When the first eight edges of EB method are removed, the connectivity is the same as that of MCCA. After removing all 15 bridge-edges, the connectivity of DS, BS, EB and MCCA are decreased by 7.7\%, 0\%, 56.41\% and 56.41\%, respectively. 


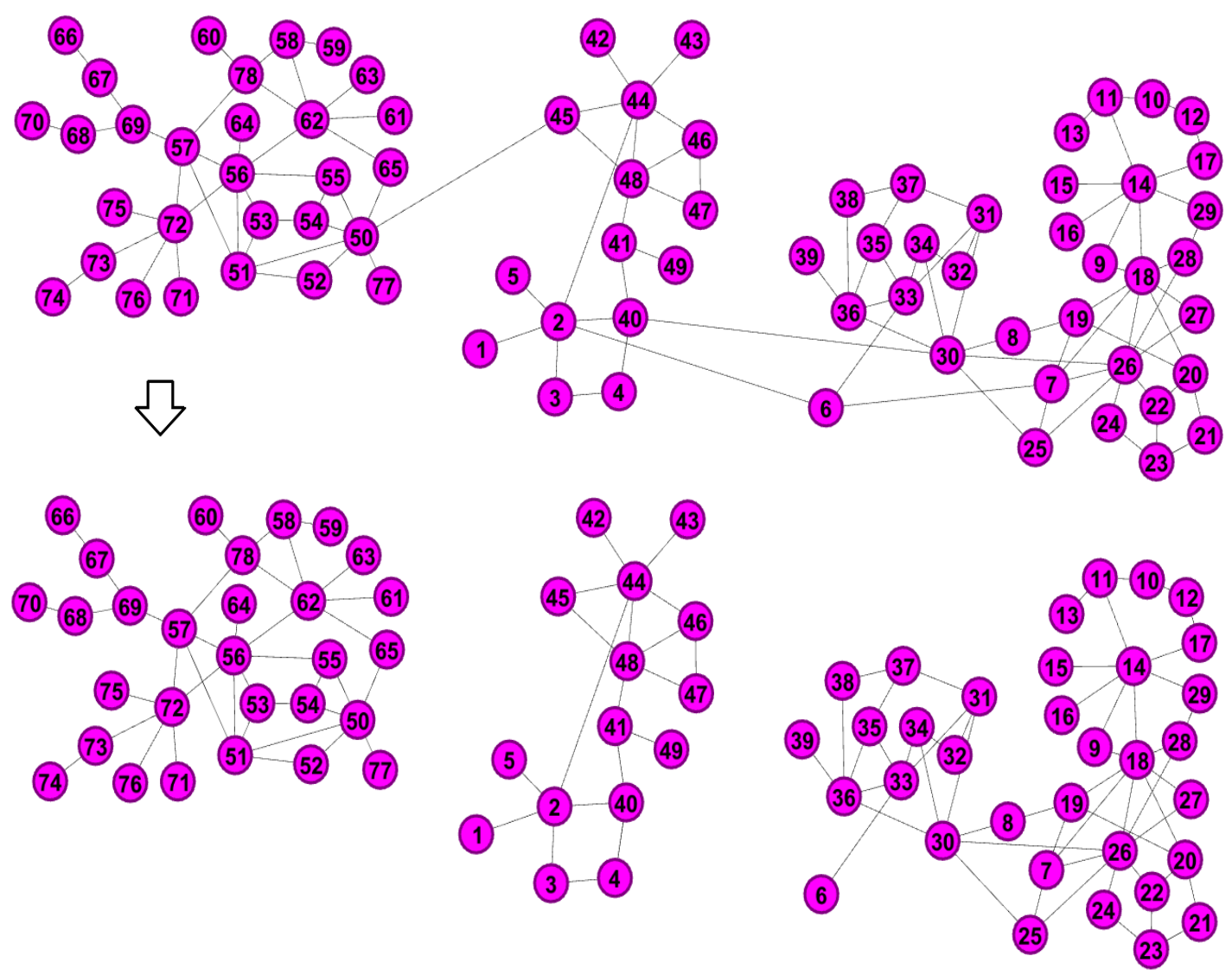

Fig. 6. The southern network before and after the first four edges removed

\section{B. Connectivity on the whole provincial network}

Fig. 7 shows the trend of network connectivity with the removal of bridge-edges in the whole provincial complex power optical cable network model.

In Fig. 7, the connectivity decreases slowly in the interval 0-7 of horizontal axis. With the increase of the number of removed edges, the network is divided into two independent subnetworks after the first eight bridge-edges is removed, and the connectivity of MCCA is decreased by $14.18 \%$. This is because the 8 th bridge-edge $\left(e_{222,227}\right)$ is the optical cable connection between two cities connecting the Changling communication station and the Tongyu communication station. Then, with the removal of the 22nd edge (edge $e_{53,79}$ ), the network is divided into four independent sub-networks, and the connectivity is decreased by $33.33 \%$. The $G_{i j}$ without the edge is shown in Fig. 8. The connectivity changes process with the 22nd edge removed is shown in Fig. 9. The connectivity of MCCA, DS, BC and EB are decreased by $51.72 \%, 24.14 \%, 24.14 \%$ and $20.69 \%$ respectively after removing all 35 bridging-edges. Compared with the other three methods, the connectivity of MCCA is increased by $27.58 \%, 27.58 \%$ and $31.03 \%$ respectively. 


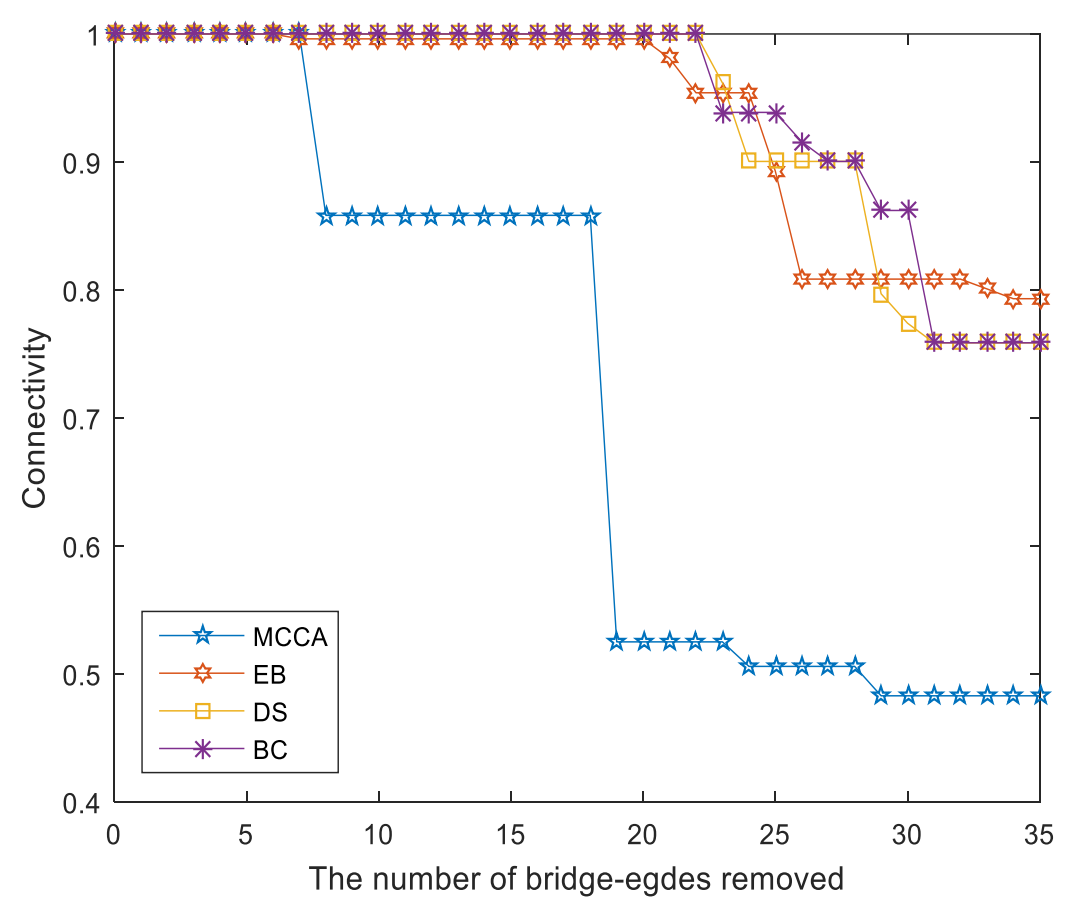

Fig. 7. The trend of network connectivity with the removal of bridge-edges

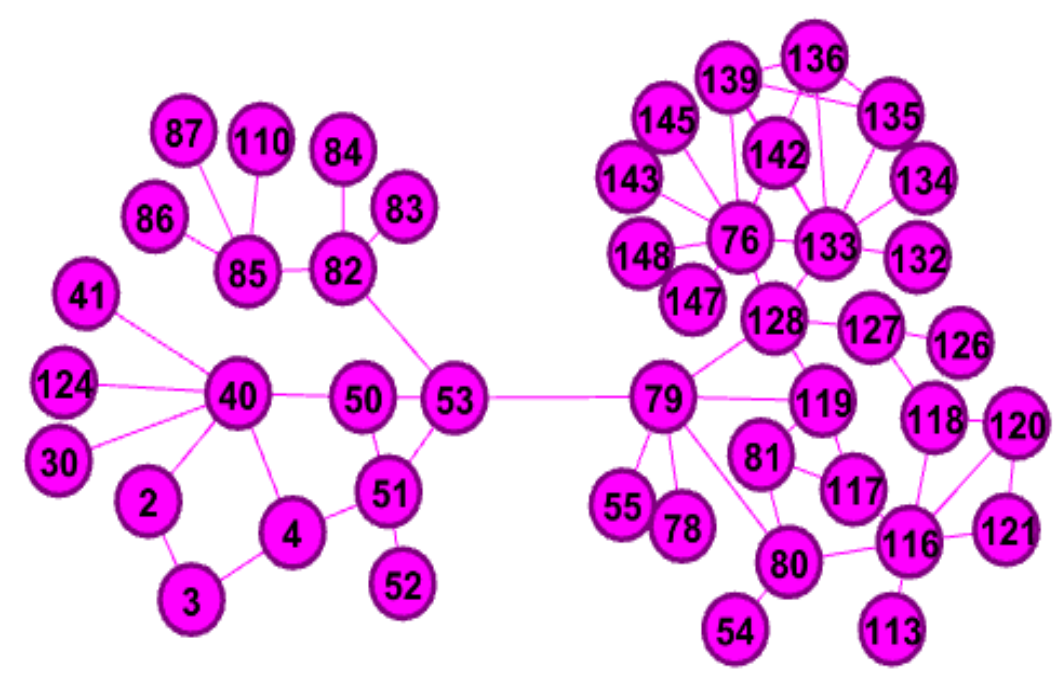

Fig. 8. The $G_{i j}$ of the 22th edge (edge $e_{53,79}$ ) 

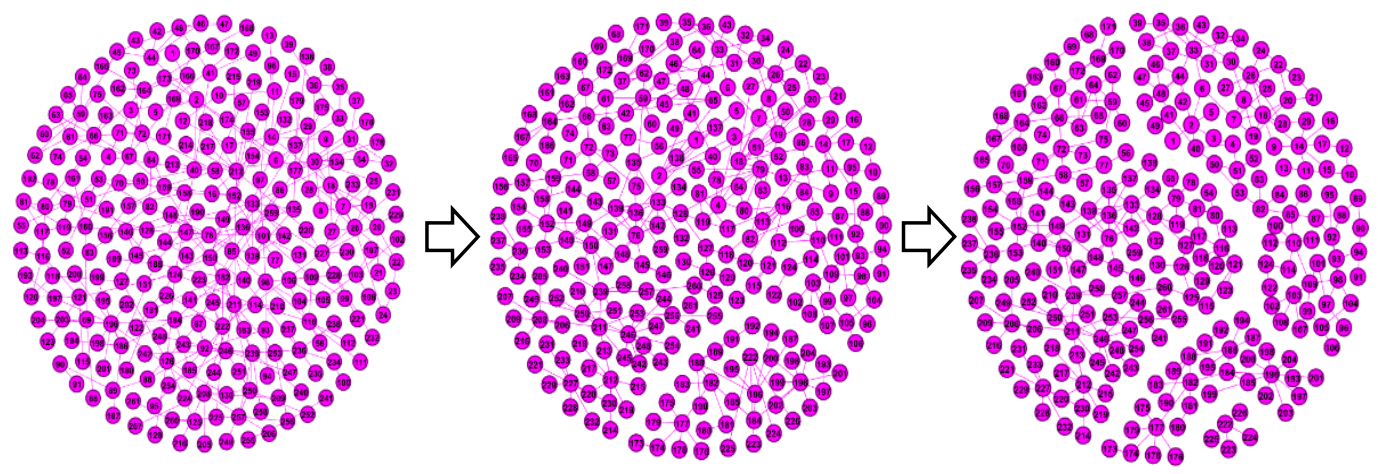

Fig. 9. The connectivity changes process of the network with the 22nd edge removed

\subsubsection{Network efficiency analysis}

After bridge-edges were mined, to verify the rationality and validity of the proposed method, the effect of bridge-edges on decline rate of network efficiency of each complex power optical cable network is analyzed.

\section{A. The decline rate of network efficiency on the southern provincial network}

Fig. 10 shows the trend of decline rate of network efficiency with the removal of bridge-edges in the southern complex power optical cable network model.

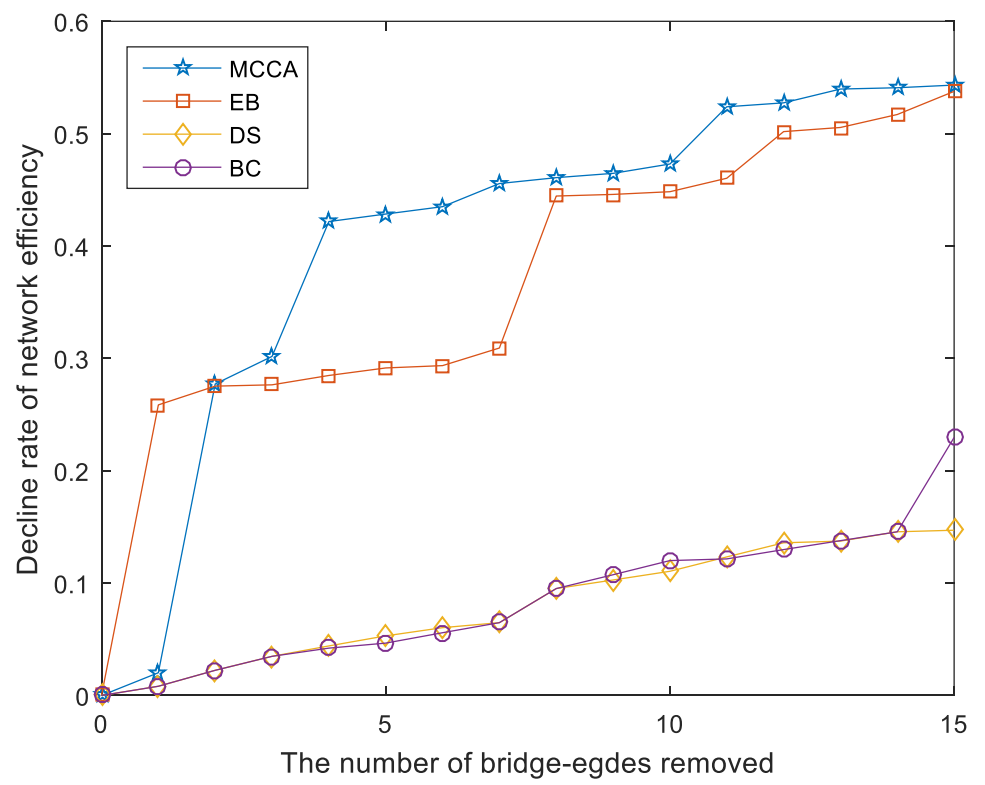

Fig. 10. The decline rates of network efficiency with the removal of bridge-edges

In Fig. 10, it shows the relationship between the decline rate of network efficiency and the number of bridge-edges removed from the network. With the removal of bridge-edges, the curve of decline rate of network efficiency show a downward trend as a whole. When the same number of edges are removed, the decline rate of network efficiency of MCCA is similar to that of EB, but higher than that of DS and BC. When all 15 edges are removed, the decline 
rate of network efficiency of MCCA, EB, DS, BC are increases by $54.32 \%, 53.77 \%, 14.71 \%$ and $22.96 \%$, respectively. The average decline rate of network efficiency of MCCA, EB, DS and $\mathrm{BC}$ are $1.55 \%, 1.54 \%, 0.42 \%$ and $0.66 \%$, respectively. Compared with the average decline rates of network efficiency of other three methods, the average decline rate of network efficiency of MCCA is the highest.

\section{B. The decline rate of network efficiency on the whole provincial network}

Fig. 11 shows the trend of decline rate of network efficiency with the removal of bridge-edges in the whole provincial complex power optical cable network model.

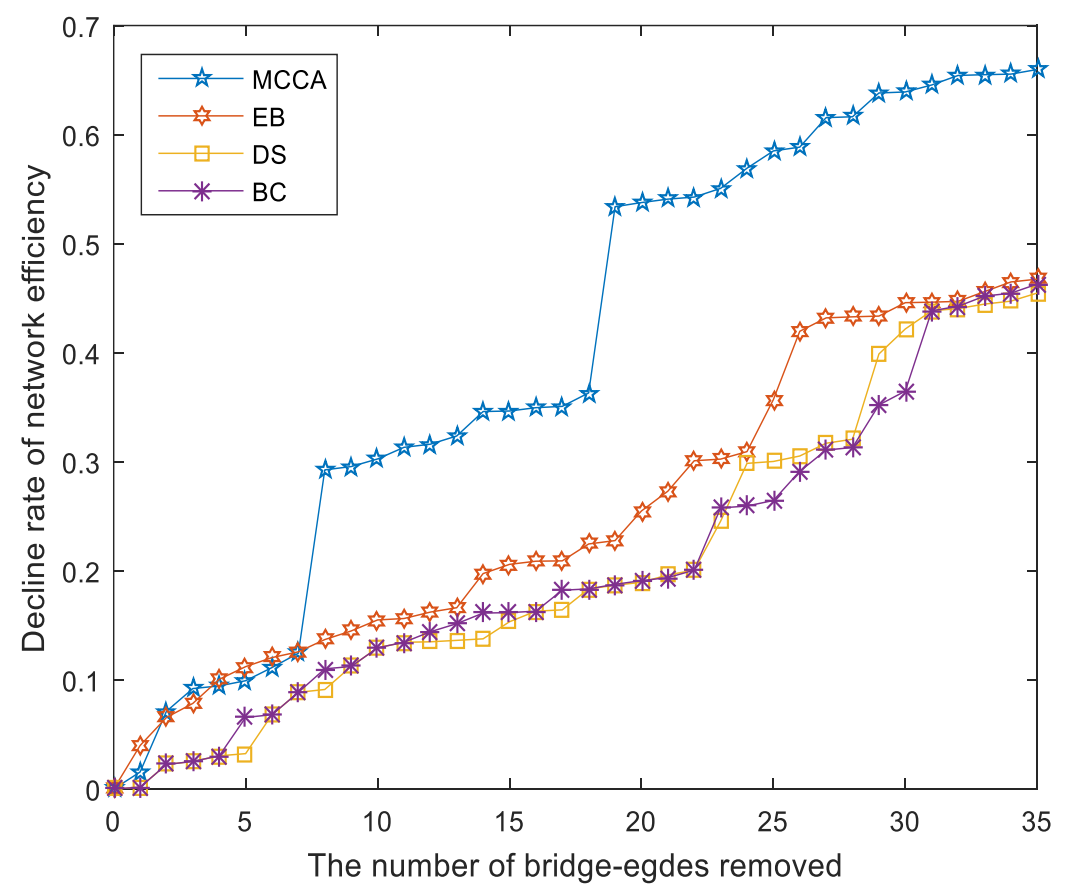

Fig. 11. The decline rate of network efficiency with the removal of bridge-edges

From the overall trend of network efficiency decline rate of the four methods in Fig. 11, it can be found that the upward trend of network efficiency decline rate of MCCA is significantly higher than that of the other three methods. When all 35 edges are removed, the decline rates of network efficiency of MCCA, EB, DS, BC are increases by $66 \%, 47 \%, 46 \%$ and $46 \%$, respectively. The average decline rates of network efficiency of MCCA, EB, DS and BC are $1.89 \%, 1.34 \%, 1.31 \%$ and $1.31 \%$, respectively. Compared with the average network efficiency decline rates of the other three methods, the average decline rates of network efficiency MCCA are increased by $0.55 \%, 0.58 \%$ and $0.58 \%$ respectively.

The above two evaluation criteria are further verified the influence of bridge-edges on network reliability from two aspects of network performance and structural stability. The verification results show that the MCCA method can effectively mine the bridge-edges in the power optical cable network. According to the experimental results, with the removal of the top $10 \%$ of the bridge-edges in the complex power optical cable network model of Jilin Province, compared with that of other three typical methods, the decline rate of network efficiency of MCCA method is increased by 19\%, $20 \%$ and $20 \%$, respectively, and the connectivity is decreased by $31.03 \%, 27.58 \%$ and $27.58 \%$. 


\section{Conclusion}

The bridge-edges play the role of connecting the communication between regions, which has a crucial impact on the reliability of power optical cable network. Therefore, to mine bridgeedges in power optical cable network, the complex power optical cable network model of the provincial power optical cable network is constructed. And to measure the generated potential energy of all nodes in n-level neighborhood local structure for the edge, the n-level neighborhood local structure topological potential is designed. Then, the minimum connected chain attenuation is designed to measure the attenuation degree caused by substituted edges. Finally, a bridge-edges mining algorithm based on the minimum connected chain attenuation topological potential is proposed. The experimental results show that, compared with the existing methods (degree summation, bridge coefficient and edge betweenness), the proposed method can effectively mine the bridge-edges of power optical cable network, not only can mine the optical cable connection between $500 \mathrm{kV}$ communication stations, but also can mine the optical cable connection with "bridge characteristic" between municipal or county-level city communication stations, and the optical cables without obvious characteristics of city or voltage, but it have "bridge characteristics" in topology structure.

The research on the reliability of complex networks is still a hot topic at present, and the research based on the bridge-edge still needs further study. In the future, we will study how to use bridge-edge to mine the backbone structure of the power optical cable network, and how to add edges to the network to reduce the impact of bridge-edge on the reliability of the power optical cable network.

\section{Acknowledgement}

This work was supported in part by the project of the National Natural Science Foundation of China under Grant 61572420, in part by Research Project of the Education Department of Jilin Province under Grant JJKH20190706KJ and in part by Science and technology innovation development program of Jilin under Grant 20190104140.

\section{References}

[1] J. Zhao, H. Zhou, B. Chen, and P. Li, "Research on the Structural Characteristics of Transmission Grid Based on Complex Network Theory," Journal of Applied Mathematics, vol. 2014, no. 8, pp. 1-12, Apr. 2014. Article (CrossRef Link)

[2] Z. Tian, L. Jia, H. Dong, F. Su, and Z. D. Zhang, "Analysis of Urban Road Traffic Network Based on Complex Network," Procedia Engineering, vol. 137, pp. 537-546, 2016. Article (CrossRef Link)

[3] F. Mao, L. Ma, Q. He, and G. Xiao, "Match Making in Complex Social Networks," Applied Mathematics and Computation, vol. 371, pp. 1-13, 2020. Article (CrossRef Link)

[4] W. Pan, B. Li, J. Liu, Y. T. Ma, and B. Hu, "Analysing the Structure of Java Software Systems by Weighted K-core,” Future Generation Computer Systems, vol. 83, pp. 431-444, 2018. Article (CrossRef Link)

[5] M. Bellingeri and D. Cassi, "Robustness of Weighted Networks," Physica A: A Statistical mechanics and its Applications, vol. 489, pp. 47-55, 2018. Article (CrossRef Link)

[6] X. Zhu, W. Song, and L. Gao, "Topological Characteristics and Vulnerability Analysis of Rural Traffic Network,” Journal of Sensors, vol. 2019, pp. 1-9, Apr. 2019. Article (CrossRef Link)

[7] Y. Lyu and P. Yin, "Internet of Things Transmission and Network Reliability in Complex Environment," Computer Communications, vol. 150, pp. 757-763, 2020. Article (CrossRef Link)

[8] S. W. Mei and X. P. Ni, "Several New Progresses on the Complexity Research of the Interconnected Power Network," Journal of Changsha University of Science and 
Technology(Natural Science), vol. 5, no. 2, pp. 4-12, 2008. Article (CrossRef Link)

[9] Z. X. Cai, X. H. Wang, and X. N. Ren, "A Review of Complex Network Theory and Its Application in Power Systems,” Power System Technology, vol. 32, no. 11, pp. 114-121, Nov. 2012. Article (CrossRef Link)

[10] Y. J. Cao, Y. D. Zhang, and Z. J. Bao, “Analysis of Cascading Failures under Interactions between Power Grid and Communication Network," Electric Power Automation Equipment, vol. 33, no, 1, pp. 7-11, Jan. 2013. Article (CrossRef Link)

[11] B. Li, J. Zhang, S. S. Chen, C. Y. Zhu, D. S. Jing, and B. Q, "Expansion Strategy of Power Communication Network Survivability Based on Complex Network," Power System Technology, vol. 42, no. 6, pp. 1974-1980, June 2018. Article (CrossRef Link)

[12] J. H. Yang, X. D. Peng, and Y. J. Chao, "Topology Analysis and Optimization of Power Communication Network Based on Complex Network," Computer \& Digital Engineering, vol. 46, no. 11, pp. 2319-2328, Nov. 2018. Article (CrossRef Link)

[13] L. Liu, Y. H. Tan, J. Y. Jin, J. Zhang, and F. G. Tong, "Key Node Identification of Power Communication Network," Proceedings of the CSU-EPSA, vol. 32, no. 2, pp. 28-34, 2019. Article (CrossRef Link)

[14] D. C. Liu, X. P. Ji, B. Wang, and F. Tang, "Topological Vulnerability Analysis and Countermeasures of Electrical Communication Network Based on Complex Network Theory," Power System Technology, vol. 39, no. 12, pp. 3615-3621, Dec. 2015. Article (CrossRef Link)

[15] Y. Zeng, Y. Wang, X. J. Dong, and B. Fan, "Node Importance Evaluation Strategy on Electric Power Communication Backbone Network," Journal of North China Electric Power University, vol. 40, no. 5, pp. 65-69, Sep. 2013. Article (CrossRef Link)

[16] Y. J. He, H. X. Gao, and S. P. Zhou, "Research on Link Importance Identification for Backbone Optical Communication Network," Study on Optical Communications, vol. 6, no. 8, pp. 52-56, Apr. 2018. Article (CrossRef Link)

[17] J. Yin, G. J. Li, and H. G. Huang, “Analysis of power telecommunication network vulnerability based on link used rate," Power System Protection and Control, vol. 46, no. 2, pp. 31-36, Jan. 2018. Article (CrossRef Link)

[18] Z. Y. Xia, D. X. Chen, L. Wei, J. Q. Shi, N. Sun, and M. Lu. "Topology Analysis and Research on the Power Fiber Transmission Network in Nanjing," Power System \& Automation, vol. 30, no. 3, pp. 60-63, Nov. 2016. Article (CrossRef Link)

[19] J. Liu, Q. Y. Xiong, W. R. Shi, X. Shi, and K. Wang, "Evaluating the Importance of Nodes in Complex Networks," Physica A: Statistical Mechanics and its Applications, vol. 452, pp. 209-219, 2016. Article (CrossRef Link)

[20] W. Liu, M. Pellegrini, and A. Wu, "Identification of Bridging Centrality in Complex Networks," IEEE Access, vol. 7, pp. 93123-93130, June 2019. Article (CrossRef Link)

[21] X. Zhang, J. Zhu, Q. Wang, and H. Zhao, "Identifying Influential Nodes in Complex Networks with Community Structure," Knowledge-Based Systems, vol. 42, pp. 74-84, 2013. Article (CrossRef Link)

[22] Y. Qiu and H. E. Xing, "Evaluation Method for Critical Edge of Operation Networks Based on Complex Theory," Ordnance Industry Automation, vo1. 30, no. 8, pp. 22-26, Aug. 2011. Article (CrossRef Link)

[23] C. S. Pan, X. S. Chen, Y. M. Wang, and B. Chen, "A Method of Key Links Identification in Command and Control Networks Based on Bridging Coefficient," in Proc. of IEEE 15th International Conference on Networking, Sensing and Control (ICNSC), pp. 1-5, Mar. 2018. Article (CrossRef Link)

[24] X. E. Gao, X. S. Chen, Y. M. Wang, and B. Chen, "Bridge-edge Identification Method of Command and Control Network Based on Bridging Coefficient," Computer Engineering, vol. 45, no.7, pp. 309-314, July 2019. Article (CrossRef Link)

[25] J. Hu, Y. N. Han, and J. Hu, "Topological Potential: Modelling Node Importance with Activity and Local Effect in Complex Networks," in Proc. of the $2^{\text {nd }}$ International Conference on Computer Modelling \& Simulation, pp. 411-415, Jan. 22-24, 2010. Article (CrossRef Link) 


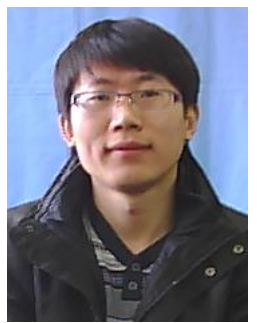

Wanchang Jiang received a B.S. degree from Liaocheng University, in 2005, and M.S. and Ph.D. degrees from Yanshan University, in 2008 and 2017, respectively. Since 2008, he has been a Teacher with the School of Computer Science, Northeast Electric Power University. Currently, he is a Associate Professor. His research interests include data mining and complex networks.

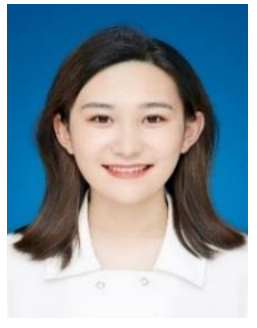

Yanhui Liu received a B.S. degree from University of Science and Technology Liaoning in 2018. She is currently pursuing a master's degree in the School of Computer Science, Northeast Electric Power University. Her main research interest is complex networks.

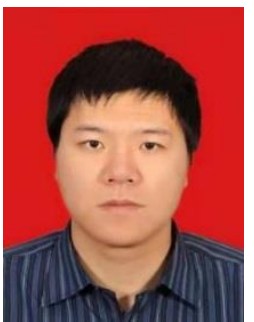

Shengda Wang received a B.S. degree from Changchun University of Science and Technology in 2005. He is currently with the Jilin Information \& Telecommunication Company, Jilin Electric Power Corporation Ltd as a Deputy Senior Engineer. His main research interest is optical fiber communication network.

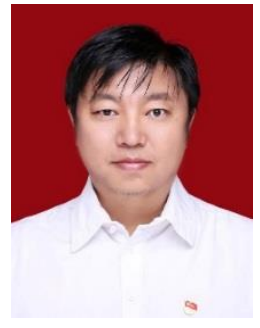

Jian Guo received a B.S. degree from Tianjin University in 1998. He has been working in Jilin Power Supply Company of State Grid since 1998 and is now the deputy manager. His main research interest is optical fiber communication network. 